SLAC-PUB-9631

August 2003

Revised

\title{
Beam Dynamics of the Interaction Region Solenoid in a Linear Collider Due to a Crossing Angle*
}

\author{
P. Tenenbaum , J. Irwin, T.O. Raubenheimer \\ Stanford Linear Accelerator Center, Stanford University, Stanford, CA
}

\begin{abstract}
Future linear colliders may require a nonzero crossing angle between the two beams at the interaction point. This requirement in turn implies that the beams will pass through the strong interaction region (IR) solenoid with an angle, and thus that the component of the solenoidal field perpendicular to the beam trajectory is nonzero. The interaction of the beam and the solenoidal field in the presence of a crossing angle will cause optical effects not observed for beams passing through the solenoid on-axis; these effects include dispersion, deflection of the beam, and synchrotron radiation effects. For a purely solenoidal field, the optical effects which are relevant to luminosity exactly cancel at the IP when the influence of the solenoid's fringe field is taken into account. Beam size growth due to synchrotron radiation in the solenoid is proportional to the fifth power of the product of the solenoidal field, the length of the solenoid, and the crossing angle. Examples based on proposed linear collider detector solenoid configurations are presented.
\end{abstract}

Published in Physical Review Special Topics - Accelerators and Beams

*Work supported by the Department of Energy, Contract DE-AC03-76SF00515. 


\title{
Beam Dynamics of the Interaction Region Solenoid in a Linear Collider Due to a Crossing Angle
}

\author{
P. Tenenbaum, ${ }^{*}$ J. Irwin, and T. O. Raubenheimer \\ Stanford Linear Accelerator Center, Stanford University, Stanford, CA 94309 USA
}

\begin{abstract}
Future linear colliders may require a nonzero crossing angle between the two beams at the interaction point. This requirement in turn implies that the beams will pass through the strong interaction region (IR) solenoid with an angle, and thus that the component of the solenoidal field perpendicular to the beam trajectory is nonzero. The interaction of the beam and the solenoidal field in the presence of a crossing angle will cause optical effects not observed for beams passing through the solenoid on-axis; these effects include dispersion, deflection of the beam, and synchrotron radiation effects. For a purely solenoidal field, the optical effects which are relevant to luminosity exactly cancel at the IP when the influence of the solenoid's fringe field is taken into account. Beam size growth due to synchrotron radiation in the solenoid is proportional to the fifth power of the product of the solenoidal field, the length of the solenoid, and the crossing angle. Examples based on proposed linear collider detector solenoid configurations are presented.
\end{abstract}

\section{INTRODUCTION}

In order to meet the goals of particle physics experimenters, a future linear collider must achieve a luminosity in excess of $10^{34} \mathrm{~cm}^{-2} \mathrm{sec}^{-1}$ and a center-of-mass energy between $500 \mathrm{GeV}$ and $5 \mathrm{TeV}$. In addition, both engineering and other limitations will prohibit such a facility from consuming more that a few hundred megawatts of electric power. Meeting the specifications of the experimenters within such a tight energy budget can only be done by accelerating a long train of bunches on each RF pulse and reducing the RMS size of the beam at the collision point to a few hundred nanometers in the horizontal and a few nanometers in the vertical. The Next Linear Collider (NLC), for example, can achieve a luminosity of $3 \times 10^{34} \mathrm{~cm}^{-2} \mathrm{sec}^{-1}$ at $1 \mathrm{TeV}$ in the center of mass by accelerating $0.75 \times 10^{10}$ particles per bunch, 192 bunches per train, 120 trains per second, and colliding 219 nanometer by 2.3 nanometer (RMS) beams at the IP. Each bunch train is 268 nanoseconds long; therefore the spacing between bunches in a train is 1.4 nanoseconds [1].

Because the beam density at the collision point is so high, the beam-beam focusing effect is very strong. As a result, the beams which emerge from the collision have a much larger angular divergence than the beams which enter the collision, and appropriate stay-clears for the outgoing beam are required. In the case of the NLC, the incoming beam divergence is approximately 20 microradians RMS in both horizontal and vertical; the stay-clear requirement for the outgoing beam is defined by a cone of "beamstrahlung" photons which contains approximately 1 megawatt of average power within an RMS angular divergence of 100 microradians (horizontal) by 50 microradians (vertical) [2]. An additional constraint on interaction region design is that parasitic collisions between the two beams must be avoided. In the case of the NLC, both of these issues - parasitic collisions and providing adequate aperture for the outgoing beam - can be addressed by introducing a small crossing angle at the IP. By colliding the beams at an angle of 20 milliradians, the transverse offset at the first parasitic collision can be made so large that the beams essentially do not influence one another, and the outgoing beam can exit through a beampipe with a larger aperture than the incoming beamline.

*quarkpt@slac.stanford.edu 
An alternate approach to managing the pathologies of high-luminosity collisions is to dramatically increase the spacing of bunches within a single train. Such a design choice permits head-on collisions if the vacuum chamber through the final lenses in the detector is large enough to accommodate the disrupted outgoing beam. For example, the TESLA superconducting linear collider design stipulates an intra-bunch spacing of 337 nanoseconds [3] and a relatively large final doublet aperture radius of 2.4 centimeters [4]. Unfortunately, such a large intra-bunch spacing is out of the question for a room-temperature linear collider. In addition, collisions at a center-of-mass energy significantly above $1 \mathrm{TeV}$ will result in substantial pair production through coherent processes. The particles generated in this way will have energies far below the energy of the beam, and they will leave the collision point with angles on the order of milliradians. It is not possible to remove these particles from the detector through the quads used to focus the incoming beams; a quad-free exit hole with an acceptance of at least several milliradians is required to safely remove both the high-energy spent beam and the low-energy pairs from the detector. Consequently, a linear collider with a center-ofmass energy over $1 \mathrm{TeV}$ will almost certainly require a crossing angle, no matter what the choice of accelerating technology [5].

If the particle physics detector is positioned symmetrically with respect to the two beams, then each beam will travel with an angle $\theta_{c}$ with respect to the detector's longitudinal axis, as shown schematically in Figure 1. As a result, the beam will pass through the detector solenoid at an angle. This has two effects: the beam has a small angle with respect to the longitudinal field of the solenoid, and the beam is far from the solenoid's axis when it passes through the fringe field at the end of the solenoid. Both of these effects cause the beam to encounter a nonzero vertical bending field. As a result, the beam is deflected vertically from its original trajectory as it passes through the solenoid. Because particles at lower energies experience a larger deflection than those that are at relatively high energies, the deflection gives rise to a vertical dispersion. Finally, the beam will emit synchrotron radiation as it is deflected. All of these effects are potentially deleterious: the deflection can cause the two beams to miss at the nominal collision point; dispersion can cause the beam size to increase; and synchrotron emission in a dispersive region can cause irreversible growth in the IP spot size. Because all of these effects operate in the vertical plane, and the vertical beam size is typically much smaller than the horizontal, the impact of the solenoid on the luminosity can be especially severe.

In this paper, we estimate the impact of these effects in the case of a beam with energy $E$, magnetic rigidity $B \rho$, and Lorentz factor $\gamma \equiv E / \mathrm{m}_{\mathrm{e}} \mathrm{c}^{2}$ as it passes through a pure solenoid with angle $\theta_{c}$ relative to the solenoid axis (thus $\theta_{c}$ is half the beam-beam crossing angle). The solenoid is assumed to have a design field of $B_{0}$ at the collision point, and the collision point occurs at the symmetry point of the solenoid. Our coordinate system is chosen such that $z \equiv 0$ is at the center of the solenoid, and $z>0$ along the path of the beam (i.e., the beam has a velocity which is negative in this coordinate system). In general we assume the particles of interest are positrons, to simplify the selection of signs. The focusing effect of the solenoid is typically quite weak for the parameters of a linear collider, and is neglected here.

\section{OPTICAL EFFECTS}

In the simplest model of a detector solenoid - in which the solenoidal field is uniform and longitudinal, $\vec{B}=B_{0} \hat{z}$, for $z<z_{\max }$ and falls instantly to zero for $z \geq z_{\max }$ - the vertical deflection of a 
positron beam at any point $z_{0} \leq z_{\max }$ is given by:

$$
\begin{aligned}
y(z) & =\int_{z}^{z_{\max }}\left(z^{\prime}-z\right) y^{\prime \prime}\left(z^{\prime}\right) d z^{\prime} \\
& =\frac{1}{2} \frac{B_{0} \sin \theta_{c}}{B \rho}\left(z_{\max }-z\right)^{2} .
\end{aligned}
$$

In the absence of transverse focusing effects, the dispersion function, $\eta_{y}(z)$, is equal and opposite to the deflection: $\eta_{y}(z)=-y(z)$.

A somewhat more accurate model of the solenoid includes the fringe field, $B_{r}(r, z)$, and a nonuniform longitudinal field $B_{z}(r, z)$. For the coordinate system and conventions used in Figure 1, in which $B_{r}$ and $B_{z}$ are both positive and $r(z) \equiv z \tan \theta_{c}$, Equation 1 can be rewritten as:

$$
y(z)=\frac{1}{B \rho} \int_{z}^{\infty}\left(z^{\prime}-z\right)\left[\sin \theta_{c} B_{z}\left(z^{\prime} \tan \theta_{c}, z^{\prime}\right)-\cos \theta_{c} B_{r}\left(z^{\prime} \tan \theta_{c}, z^{\prime}\right)\right] d z^{\prime} .
$$

Equation 1 shows that the fringe field acts to reduce the total deflection of the beam. In fact, the deflections due to the fringe field and the longitudinal field of the solenoid will exactly cancel at the collision point if the beam enters the solenoid through the "endcap" and not the "barrel" (i.e., the beampipe does not pass between the windings of the solenoid).

\section{A. Cancellation of the Deflection}

Conceptually, the simplest refinement to the "hard-edged" solenoid model which includes the solenoid's fringe field is shown in Figure 2: as before, the magnetic field is uniform and longitudinal, $\vec{B}=B_{z} \hat{z}$ for $z<z_{0}$ and $\vec{B}=0$ for $z>z_{0}$; the one change from the "hard-edged" model is a radial field with magnitude $B_{r}(r, z)$ which is confined to a region of length $d z$ at $z=z_{0}$. The integral form of Gauss' law of magnetostatics can be applied to a cylindrical pillbox at $z=z_{0}$, with length $d z$ and radius $r$ (henceforth the pillbox shall be referred to as volume $V$ ). Gauss' law in this case requires that:

$$
\int_{0}^{2 \pi} d \psi \int_{0}^{r} d r^{\prime} r^{\prime} B_{z}\left(r^{\prime}, \psi, z_{0}-d z / 2\right)=\int_{0}^{2 \pi} d \psi r d z B_{r}\left(r, \psi, z_{0}\right)
$$

where we have used $\psi$ as the azimuthal variable. By construction, $B_{z}$ is a constant at all points between $z=0$ and $z=z_{0}$, so the left hand side of Equation 3 is easily evaluated. If we require that $V$ be centered on the axis of the solenoid, then $B_{r}$ is required to have azimuthal symmetry, which permits the integral on the right hand side of Equation 3 to be completed by inspection. Thus,

$$
\pi r^{2} B_{z}=2 \pi r d z B_{r}\left(r, z_{0}\right)
$$

By cancelling common factors and substituting a value of $z_{0} \tan \theta_{c}$ for $r$, we can find a useful relation between the radial field and the longitudinal field experienced by a beam passing through the solenoid with an angle $\theta_{c}$ relative to the axis:

$$
B_{r}\left(r=z_{0} \tan \theta_{c}, z_{0}\right) d z=\frac{1}{2} z_{0} \tan \theta_{c} B_{z} .
$$

We can now evaluate the deflection of the beam at the IP resulting from the radial field at $z=z_{0}$ and the corresponding longitudinal field which fills the space from $z=0$ to $z=z_{0}$. We can rewrite 
Equation 2:

$$
y(z=0)=\frac{1}{B \rho}\left[\int_{z=0}^{z=z_{0}} d z^{\prime} z^{\prime} \sin \theta_{c} B_{z}-z_{0} \cos \theta_{c} B_{r}\left(r=z_{0} \tan \theta_{c}, z_{0}\right) d z\right] .
$$

Equation 6 can be simplified by using Equation 5 to eliminate $B_{r}\left(r=z_{0} \tan \theta_{c}, z_{0}\right) d z$ from the second term on the right, and by moving constants out of the integral in the first term on the right:

$$
\begin{aligned}
y(z=0) & =\frac{1}{B \rho}\left[\sin \theta_{c} B_{z} \int_{z=0}^{z=z_{0}} d z^{\prime} z^{\prime}-\frac{1}{2} z_{0}^{2} \cos \theta_{c} \tan \theta_{c} B_{z}\right] \\
& =\frac{1}{B \rho}\left[\frac{1}{2} z_{0}^{2} \sin \theta_{c} B_{z}-\frac{1}{2} z_{0}^{2} \sin \theta_{c} B_{z}\right] \\
& =0 .
\end{aligned}
$$

We can extend our result from this somewhat idealized situation to a real detector solenoid by noting that the real radial field of the solenoid along the beam trajectory, $B_{r}\left(r=z \tan \theta_{c}, z\right)$, can be expressed as a superposition of longitudinal slices of the form described above. Each longitudinal slice of radial field is accompanied by a change in the longitudinal magnetic field which satisfies Equation 5 ; the combination of the radial field and the change in the longitudinal field will cancel for each slice of radial field, thus the total deflection from all slices is also zero. Note that if the beampipe passes between the windings of the solenoid, one of the conditions of the preceding derivation (specifically, a uniform value of $B_{z}$ downstream of the slice of radial field) cannot be satisfied and therefore the derivation holds only for beams which enter the solenoid through the "endcap" region.

In an actual linear collider installation, the ideal solenoidal field depicted above will not be achievable and therefore some residual offset will be present at the IP. Effects which will break the required symmetries include dipole steering magnets in the solenoid, insertion of quadrupoles into the solenoid, and asymmetries in the detector endcaps. The steering from these perturbations is nonetheless much smaller than what would be expected in the case where the cancellation between the fringe and longitudinal fields is not taken into account.

\section{B. A Note on Colliding Beams}

The calculations above have all shown the dynamical effects of the solenoid on one beam entering from $z>0$ and approaching the IP. For the purposes of luminosity production, the interaction of the other beam must be considered as well.

The colliding-beam case is shown in Figure 3. The symmetries of the solenoid dictate that the fringe field on the $z<0$ side of the detector will have the same effect as the fringe field on the $z>0$ side for beams of like charge. Thus, if the fringe field on the $z>0$ side of the detector deflect positron beams down and electron beams up, the fringe field on the $z<0$ side will also deflect positron beams down and electron beams up. The longitudinal field obeys the same symmetry.

In the case in which positrons enter from $z>0$ and electrons from $z<0$, and assuming a field map as shown in Figure 4, the positrons will be deflected down and back up, the electrons will be deflected up and then down, and the beams will collide at $z=0$. In this case, each beam has a vertical angle with respect to the solenoid's axis, but the beams collide head-on - their angle with respect to one another is zero. If instead the collision is electron-electron, both beams will be bent up and then down to collide, but in this case the beams will collide with a vertical crossing angle. In the case considered here, the crossing angle is 68 microradians for $1 \mathrm{TeV} \mathrm{CM}$, and is inversely 
proportional to the beam energy. Since the vertical diagonal of the beam, $\sigma_{y} / \sigma_{z}$, is approximately 21 microradians for the NLC's 1 TeV CM parameters, a vertical crossing angle of 68 microradians will result in a reduction in luminosity unless a vertical crab cavity or other mitigating technology is used.

\section{SYNCHROTRON RADIATION EFFECTS}

The deflection of the beam by the field of the solenoid will result in the emission of synchrotron radiation. This emission will result in a reduction of the average energy of the beam as it travels to the IP, and will also result in an increase in the RMS energy spread of the beam.

\section{A. Average Energy Loss}

The principal optical effect of the average beam energy loss is that the cancellation of the fringe field and the longitudinal field of the solenoid is no longer complete: the longitudinal field will deflect the beam more than the fringe field, resulting in a nonzero vertical offset and dispersion at the collision point.

How severe an effect is the average energy loss? The fractional energy loss per unit length is given by $[6]$ :

$$
\begin{aligned}
\frac{d \delta}{d s} & =\frac{C_{\gamma}}{2 \pi} \frac{E^{3}}{\rho^{2}}, \text { where } \\
C_{\gamma} & =\frac{4 \pi}{3} \frac{r_{e}^{2}}{\left(m_{e} c^{2}\right)^{3}} .
\end{aligned}
$$

If we restrict our consideration to the influence of the solenoid's strong longitudinal field, Equation 8 can be rewritten and expressed in more conventional units as:

$$
\frac{d \delta}{d s} \approx 1.3 \times 10^{-6} E[\mathrm{GeV}] \mathrm{B}_{\mathrm{z}}^{2}\left[\mathrm{~T}^{2}\right] \sin ^{2} \theta_{\mathrm{c}}
$$

As an example, consider a $5 \mathrm{~T}$ field which is uniform over 3 meters in $s$, a 10 milliradian crossing angle, and a $500 \mathrm{GeV}$ beam energy. Equation 9 indicates that the average fractional energy loss of the beam will be less than 5 parts per million. The resulting deflection of the beam at the collision point will be on the order of 2 nanometers. Such an offset can easily be corrected with a fairly weak steering dipole.

\section{B. RMS Energy Loss}

A much more interesting problem is the RMS energy loss within the solenoid. As discussed in previously, the vertical dispersion is nonzero throughout the bulk of the solenoid. The stochastic emission of synchrotron radiation in a region with vertical dispersion will cause an irreversible increase in the beam size at the collision point.

The increase in beam size at the collision point can be determined by computing the RMS increase in energy spread and the $R_{36}$ transport matrix element to the collision point at each point throughout 
the solenoid, and adding these contributions in quadrature. The mean-squared increase in relative energy spread due to synchrotron radiation is given by [7]:

$$
\sigma_{\delta}^{2}(z)=\frac{55}{24 \sqrt{3}} \frac{r_{e} \lambda_{e} \gamma^{5}}{\left|\rho^{3}(z)\right|} d z
$$

where $r_{e}$ is the classical electron radius of $2.8 \times 10^{-15}$ meters, $\lambda_{e}$ is the electron Compton radius of $3.9 \times 10^{-13}$ meters, and $\rho$ is the instantaneous bending radius. The $R_{36}$ from the point $s$ to the IP is given by:

$$
R_{36}(z \rightarrow 0)=-\int_{0}^{z} z^{\prime} d z^{\prime} \frac{B_{\perp}\left(z^{\prime}\right)}{B \rho}
$$

where we define $B_{\perp}(z)$ to be the component of the solenoidal field which is perpendicular to the beam trajectory at $z: B_{\perp}(z) \equiv \sin \theta_{c} B_{z}\left(\theta_{c} z, z\right)-\cos \theta_{c} B_{r}\left(\theta_{c} z, z\right)$. If we further define $C_{\delta} \equiv \frac{55}{24 \sqrt{3}} \frac{r_{e} \lambda_{e}}{\left(m_{e} c^{2}\right)^{5}} \approx$ $4.1 \times 10^{-11} \mathrm{~m}^{2} / \mathrm{GeV}^{5}$, we can write:

$$
\left(\Delta \sigma_{y}^{*}\right)^{2}=C_{\delta} \int_{0}^{\infty} d z \frac{E^{5}}{\left|\rho^{3}(z)\right|}\left[\int_{0}^{z} d z^{\prime} z^{\prime} \frac{B_{\perp}\left(z^{\prime}\right)}{B \rho}\right]^{2} .
$$

Equation 12 can be written in a more convenient form by replacing $1 / \rho(z)$ with $B_{\perp}(z) / B \rho$ :

$$
\left(\Delta \sigma_{y}^{*}\right)^{2}=C_{\delta} \int_{0}^{\infty} d z \frac{\left|B_{\perp}^{3}(z)\right| E^{5}}{(B \rho)^{3}}\left[\int_{0}^{z} d z^{\prime} z^{\prime} \frac{B_{\perp}\left(z^{\prime}\right)}{B \rho}\right]^{2} .
$$

Equation 13 can be further simplified by making the following 4 changes:

- Assume that $\theta_{c}$ is small and replace $\sin \theta_{c}$ with $\theta_{c}$ and $\cos \theta_{c}$ with 1 in the definition of $B_{\perp}$.

- Define dimensionless parameter $b(z)$ such that $b(z) B_{0} \theta_{c}=B_{\perp}(z)$

- Perform the outer integral from $z=0$ to $z=z_{\max }$, where $z_{\max }$ is defined to be the value of $z$ for which the solenoid's effects are reduced to negligible levels and $R_{36}\left(z_{\max } \rightarrow 0\right) \equiv 0$

- Introduce dimensionless parameter $u$ such that $u(z) z_{\max }=z$.

With these changes, Equation 13 becomes:

$$
\left(\Delta \sigma_{y}^{*}\right)^{2}=C_{\delta}\left(B_{0} \theta_{c} z_{\max }\right)^{5}\left(\frac{E}{B \rho}\right)^{5} \int_{u=0}^{u=1} d u\left|b^{3}(u)\right|\left[\int_{u^{\prime}=0}^{u^{\prime}=u} d u^{\prime} u^{\prime} b\left(u^{\prime}\right)\right]^{2} .
$$

In Equation 14, all of the dimensionful parameters that set the scale of the beam size dilution are outside of the integrals, while the two integrals are over dimensionless parameters that relate only to the shape of the solenoid's field, not its magnitude. Note also that the beam size growth does not depend upon the beam energy, since each factor of $E$ is cancelled by a factor of $B \rho$, which is linearly proportional to $E$. Given this, we can re-express Equation 14 in MKS units as:

$$
\left(\Delta \sigma_{y}^{*}\right)^{2}\left[\mathrm{~m}^{2}\right] \approx 1.0 \times 10^{-13}\left(B_{0} \theta_{c} z_{\max }\right)^{5}\left[(\text { T.m.rad })^{5}\right] \int_{u=0}^{u=1} d u\left|b^{3}(u)\right|\left[\int_{u^{\prime}=0}^{u^{\prime}=u} d u^{\prime} u^{\prime} b\left(u^{\prime}\right)\right]^{2} .
$$

As an example, consider the solenoidal field shown in Figure 4. This field map was produced for an early version of the Linear Collider Detector (LCD) design, and corresponds to a value of $\theta_{c}$ of 
$10 \mathrm{mrad}$. Figure 5 shows as functions of $u$ : the normalized deflecting field, $b(u)(\mathrm{a})$; the dispersion integral, $I_{\eta}(u) \equiv\left[\int_{u^{\prime}=0}^{u^{\prime}=u} d u^{\prime} u^{\prime} b\left(u^{\prime}\right)\right]^{2}(\mathrm{~b})$; the complete value of the outer integrand, $I(u)=|b(u)|^{3} I_{\eta}(u)$ (c). The value of the complete integral, $\int_{u=0}^{u=1} d u I(u)$, is $7.9 \times 10^{-5}$ for this example. Thus, for a 6 T solenoid, a $10 \mathrm{mrad}$ crossing angle, and a value of $z_{\max }$ of 3.9 meters, Equation 15 indicates that synchrotron radiation introduces a growth in the beam size of 0.074 nanometers, which is added in quadrature with the nominal beam size (typically $2 \mathrm{~nm}$ for $1 \mathrm{TeV}$ center-of-mass linear collider designs).

Although $0.074 \mathrm{~nm}$ is a negligible contribution to the beam size at the collision point in all linear collider designs under consideration, the rapid scaling of the beam size growth with the solenoidal field, crossing angle, and solenoid longitudinal size must not be underestimated. If, for example, the solenoid was doubled in length, the resulting beam size growth would increase to $0.42 \mathrm{~nm}$, which begins to be a significant contribution to the total beam size.

\section{RMS Energy Loss in Misaligned Solenoid}

The preceding calculation assumed that the beam trajectory is perfectly aligned with respect to the solenoid's symmetry axis. In an actual system, the solenoid and the accelerator axis are likely to be misaligned with respect to one another in the horizontal plane. This means that the beam will encounter a stronger radial field than would be expected for perfect alignment, which in turn means that the synchrotron radiation growth of the beam size will be enhanced.

A reasonably accurate estimate of the beam size growth for a horizontally misaligned solenoid can be obtained by using Equation 13, and replacing $B_{\perp}(z)$ with $B_{z}(z) \sin \theta_{c}-B_{r}(z) \cos \theta_{c}[1+$ $\Delta x /\left(z \sin \theta_{c}\right)$ ], where $\Delta x$ is the expected misalignment. It is helpful to first obtain a closed-form, upper-bound estimate on the degree of spot size dilution that can be expected from a horizontal misalignment.

Inspection of Figure 5 allows us to formulate a few simplifying assumptions that will permit a conservative estimate of the effect of a horizontal misalignment:

- Assume that the radial field's contribution to the spot size increase comes entirely from a limited region of the solenoid, from $u=u_{1}$ to $u=u_{2}$ (for example, in Figure $5(\mathrm{c}), u_{1} \approx 0.65$ and $\left.u_{2} \approx 0.77\right)$

- Assume that for $u_{1}<u<u_{2}$, the $B_{z}$ contribution to $B_{\perp}$ is negligible

- Assume that for $u_{1}<u<u_{2}$, the value of the dispersion integral $\left[\int d z^{\prime} z^{\prime} B_{\perp}\left(z^{\prime}\right)\right]^{2}$ is dominated by the design value of $B_{\perp}$.

With these assumptions, the expression for the beam size dilution from synchrotron radiation in the region from $u_{1}$ to $u_{2}$ is given by:

$$
\left(\Delta \sigma_{y}^{*}\right)_{u_{1}, u_{2}}^{2} \approx \frac{C_{\delta} E^{5}}{B \rho^{5}} \int_{u=u_{1}}^{u=u_{2}} d z\left|B_{r}(z) \cos \theta_{c}\left(1+\frac{\Delta x}{z \sin \theta_{c}}\right)\right|^{3}\left[\int_{0}^{z} d z^{\prime} z^{\prime} \frac{B_{\perp}\left(z^{\prime}\right)}{B \rho}\right]^{2}
$$

where $B_{r}$ and $B_{\perp}$ are the magnetic field values for a perfectly-aligned solenoid. If $u_{2}-u_{1}$ is small compared to the average of $u_{1}$ and $u_{2}$, we can rewrite Equation 16 as follows:

$$
\left(\Delta \sigma_{y}^{*}\right)_{u_{1}, u_{2}}^{2} \approx \frac{C_{\delta} E^{5}}{B \rho^{5}}\left(1+\frac{\Delta x}{z_{\max } \tilde{u} \sin \theta_{c}}\right)^{3} \int_{u=u_{1}}^{u=u_{2}} d z\left|B_{r}(z) \cos \theta_{c}\right|^{3}\left[\int_{0}^{z} d z^{\prime} z^{\prime} \frac{B_{\perp}\left(z^{\prime}\right)}{B \rho}\right]^{2}
$$


where $\tilde{u} \equiv\left(u_{1}+u_{2}\right) / 2$ is the center of the region that is producing the synchrotron radiation beam size growth. The integrand in Equation 17 can be recognized as the integrand that would be required to evaluate the beam size growth from $u_{1}$ to $u_{2}$ in the absence of a misalignment. We can therefore state that the misaligned solenoid will give an increase in the beam size growth given by:

$$
\Delta\left(\Delta \sigma_{y}^{*}\right)_{u_{1}, u_{2}}^{2} \approx\left(\Delta \sigma_{y}^{*}\right)_{u_{1}, u_{2}}^{2}\left[3 \frac{\Delta x}{z_{\max } \tilde{u} \sin \theta_{c}}+3\left(\frac{\Delta x}{z_{\max } \tilde{u} \sin \theta_{c}}\right)^{2}+\left(\frac{\Delta x}{z_{\max } \tilde{u} \sin \theta_{c}}\right)^{3}\right],
$$

where $\left(\Delta \sigma_{y}^{*}\right)_{u_{1}, u_{2}}^{2}$ is the increase in the spot size due to the fringe field between $u=u_{1}$ and $u=u_{2}$ for a perfectly-aligned solenoid.

We can make a further improvement in Equation 18 by noting that, by definition, $\tilde{u} \leq 1$, and that, for a perfectly aligned solenoid, the increase in the spot size due to the field between $u=u_{1}$ and $u=u_{2}$ must be less than or equal to the increase over the entire solenoid. Thus,

$$
\frac{\Delta\left(\Delta \sigma_{y}^{*}\right)_{u_{1}, u_{2}}^{2}}{\left(\Delta \sigma_{y}^{*}\right)^{2}}<\left[3 \frac{\Delta x}{z_{\max } \sin \theta_{c}}+3\left(\frac{\Delta x}{z_{\max } \sin \theta_{c}}\right)^{2}+\left(\frac{\Delta x}{z_{\max } \sin \theta_{c}}\right)^{3}\right] .
$$

As an example, consider the field map in Figures 4 and 5: since $z_{\max }=4 \mathrm{~m}$, and $\theta_{c}=10 \mathrm{mrad}$, a 1 centimeter horizontal misalignment will increase the value of $\left(\Delta \sigma_{y}^{*}\right)^{2}$ by $\left(3 \times 1 / 4+3 \times(1 / 4)^{2}+(1 / 4)^{3}\right.$, or $95 \%$. The $0.074 \mathrm{~nm}$ which is added in quadrature for a perfectly-aligned solenoid increases by almost $\sqrt{2}$, to $0.10 \mathrm{~nm}$. This is still a tolerable spot size growth, but the additional growth from the solenoid misalignment will grow rapidly with $\Delta x$. Furthermore, a solenoid misalignment as large as 1 centimeter is considered highly unlikely given modern techniques for fiducialization and alignment; a more realistic value is on the order of $1 \mathrm{~mm}$ [8].

\section{CONCLUSIONS}

The solenoidal field surrounding the collision point of a linear collider will cause a vertical deflection of the beams if the collider employs a horizontal crossing angle. The deflections can result in a net offset of the beams at the collision point, beam size growth due to vertical dispersion, and beam size growth due to synchrotron radiation.

Because of the azimuthal symmetry of the detector solenoid, the optical effects from the crossing angle - vertical offset and dispersion - will vanish at the collision point. This is due to the cancellation of the deflections introduced by the solenoid's longitudinal field and its radial fringe field.

Synchrotron radiation emission will break the symmetry that causes the solenoid's vertical deflections to cancel, but this effect is a rather weak one and will typically cause only a few nanometers' static offset of the colliding beams; this can easily be corrected using weak dipole steering magnets. A more significant effect is the beam size growth due to RMS energy loss from synchrotron radiation. This causes a growth in the mean-squared beam size which grows as the fifth power of the solenoidal field, the crossing angle, and the length of the solenoid, and which is independent of the beam energy. In the case of a realistic model of a next-generation linear collider detector field, the resulting spot size growth was found to be less than $0.1 \mathrm{~nm}$ added in quadrature with the nominal RMS vertical beam size of approximately $2 \mathrm{~nm}$. The synchrotron radiation growth is enlarged if the solenoid is horizontally misaligned with respect to the beam trajectory, but conservative estimates of the size of the effect indicate that centimeter-scale misalignments are tolerable.

In the case of a horizontally misaligned solenoid, the beam will experience a vertical deflection at the IP. The vertical offset is linearly proportional to the horizontal misalignment; in the case 


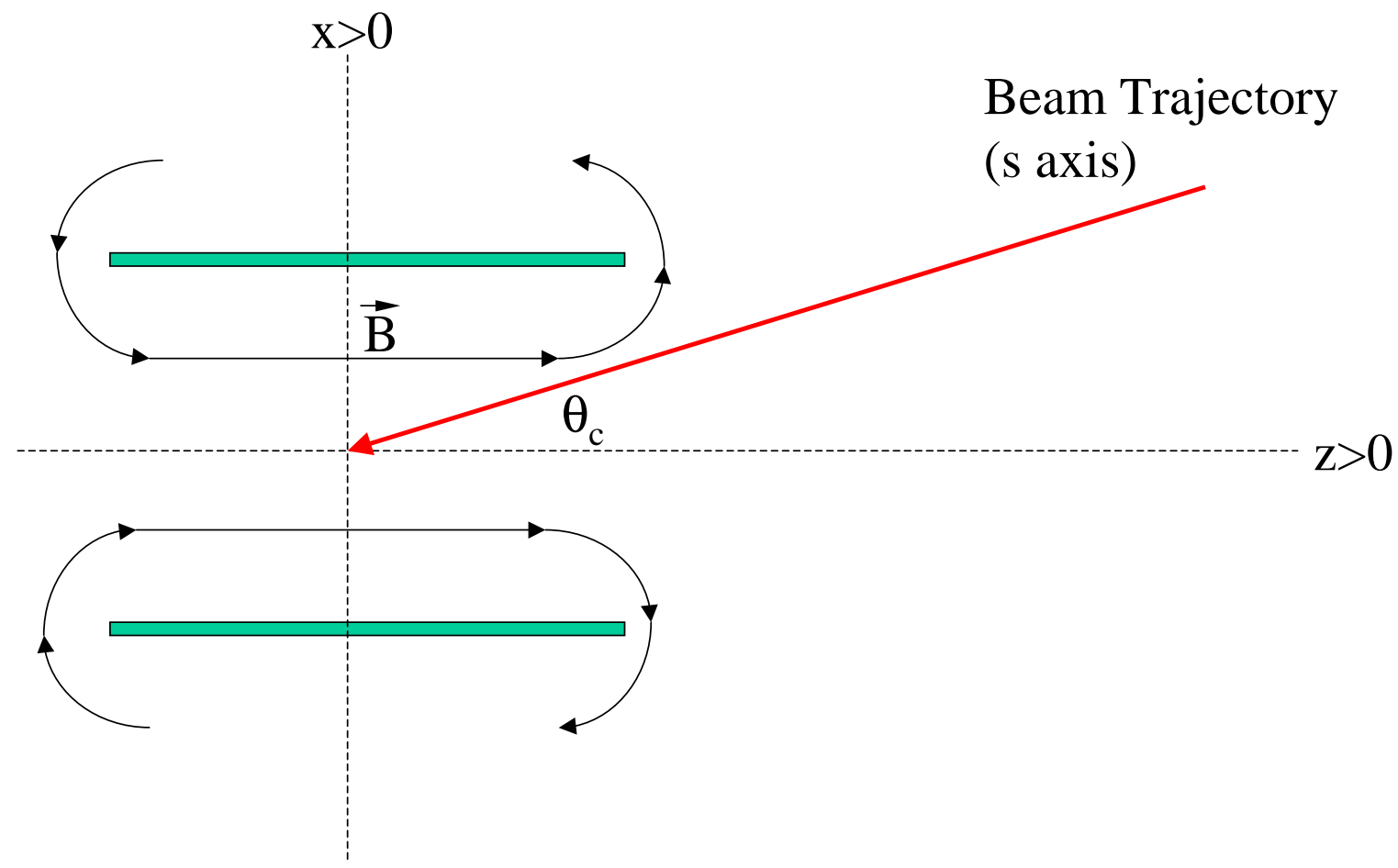

FIG. 1: (color) Plan view schematic of beam passing through a solenoid with a horizontal crossing angle.

considered, the constant of proportionality was 1/295. Unlike the other effects of the solenoid considered here, this source of vertical deflection is present even for designs which forego a crossing angle, and becomes more severe at lower beam energies.

None of these effects are expected to pose a serious limitation to a linear collider operating in the center-of-mass energy regime of 0.5 to $1.0 \mathrm{TeV}$ with a peak luminosity on the order of $10^{34} \mathrm{~cm}^{-2} \mathrm{sec}^{-1}$. For operation at much lower energy it may be necessary to reduce the strength of the detector solenoid to limit the coupling of horizontal jitter of the beam or solenoid to the vertical beam position. Given the inexorable demands for more capable detectors with higher solenoid fields, and simultaneous requirements for higher luminosity and smaller vertical beam sizes, the beam size growth from synchrotron radiation may begin to play a role in a more futuristic linear collider than those presently under consideration.

In a realistic linear collider interaction region, the final quadrupole lenses will typically protrude into the field of the solenoid some extent. This will introduce perturbations to the expressions used for estimating the optical and quantum-emission effects of the solenoid; the degree of perturbation is a strong function of the detailed geometry of the interaction region (depth of penetration of the magnets into the solenoid, use of superconducting or permanent magnets for the quads, etc.), and each design will require careful individual study and optimization. Nonetheless, the low severity of the phenomena surveyed here suggests that the system will remain tractable even when the effects of IR quadrupoles are included.

\section{Acknowledgments}

The authors would like to thank J. Hodgson of the Stanford Linear Accelerator Center for providing the LCD field maps used in the synchrotron radiation example. This work was performed under 


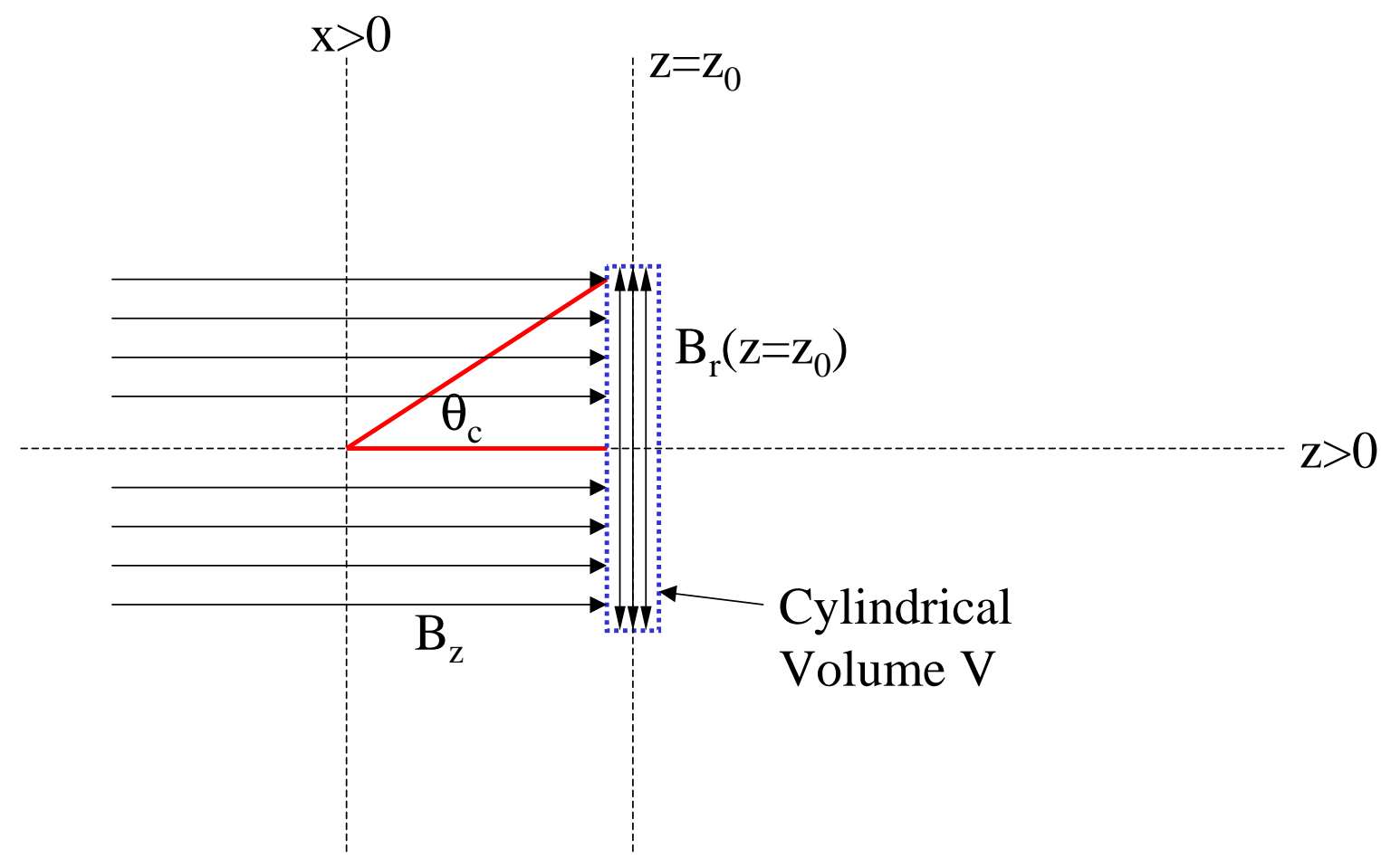

FIG. 2: (color) Beam passing through an idealized solenoid consisting of a uniform longitudinal magnetic field $B_{z}$ which drops abruptly to zero at $z=z_{0}$, and a radial field of infinitessimal length at $z=z_{0}$. The cylindrical Gaussian volume $\mathrm{V}$, as defined in the text, is shown.

Department of Energy contract DE-AC03-76SF00515.

[1] N. Phinney, NLC News 2, 1 (2001).

[2] Y. Nosochkov, T. O. Raubenheimer, K. Thompson, and M. Woods, in Proceedings of the 1999 Particle Accelerator Conference (1999), pp. 3465-3467.

[3] F. Richard, J. Schneider, D. Trines, and A. Wagner, eds., TESLA Technical Design Report (Deutsches ElektronenSynchrotron DESY, 2001), vol. 1, p. 34.

[4] R. Brinkmann, K. Flottmann, J. Rossbach, P. Schmuser, N. Walker, and H. Weise, eds., TESLA Technical Design Report (Deutsches Elektronen-Synchrotron DESY, 2001), vol. 2, p. 200.

[5] D. Schulte and F. Zimmermann, in Proceedings of the 2001 Particle Accelerator Conference (2001), pp. 148-150.

[6] H. Wiedemann, Particle Accelerator Physics (Springer-Verlag, 1993), vol. 1, p. 309.

[7] K. Oide, Physical Review Letters 61, 1713 (1988).

[8] M. Breidenbach (2003), private communication. 


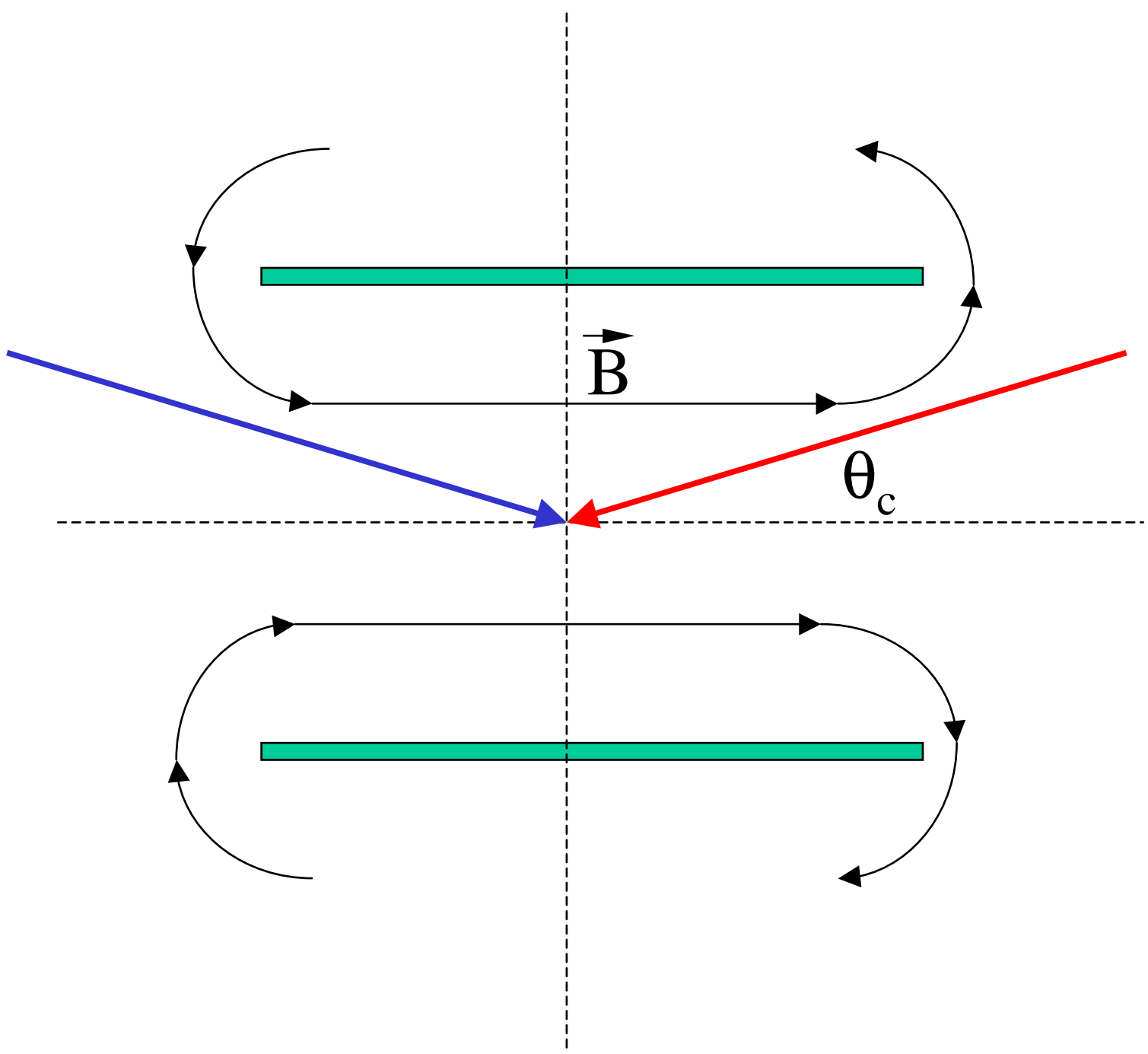

FIG. 3: (color) Schematic of 2 beams colliding with a crossing angle in the detector solenoid. 

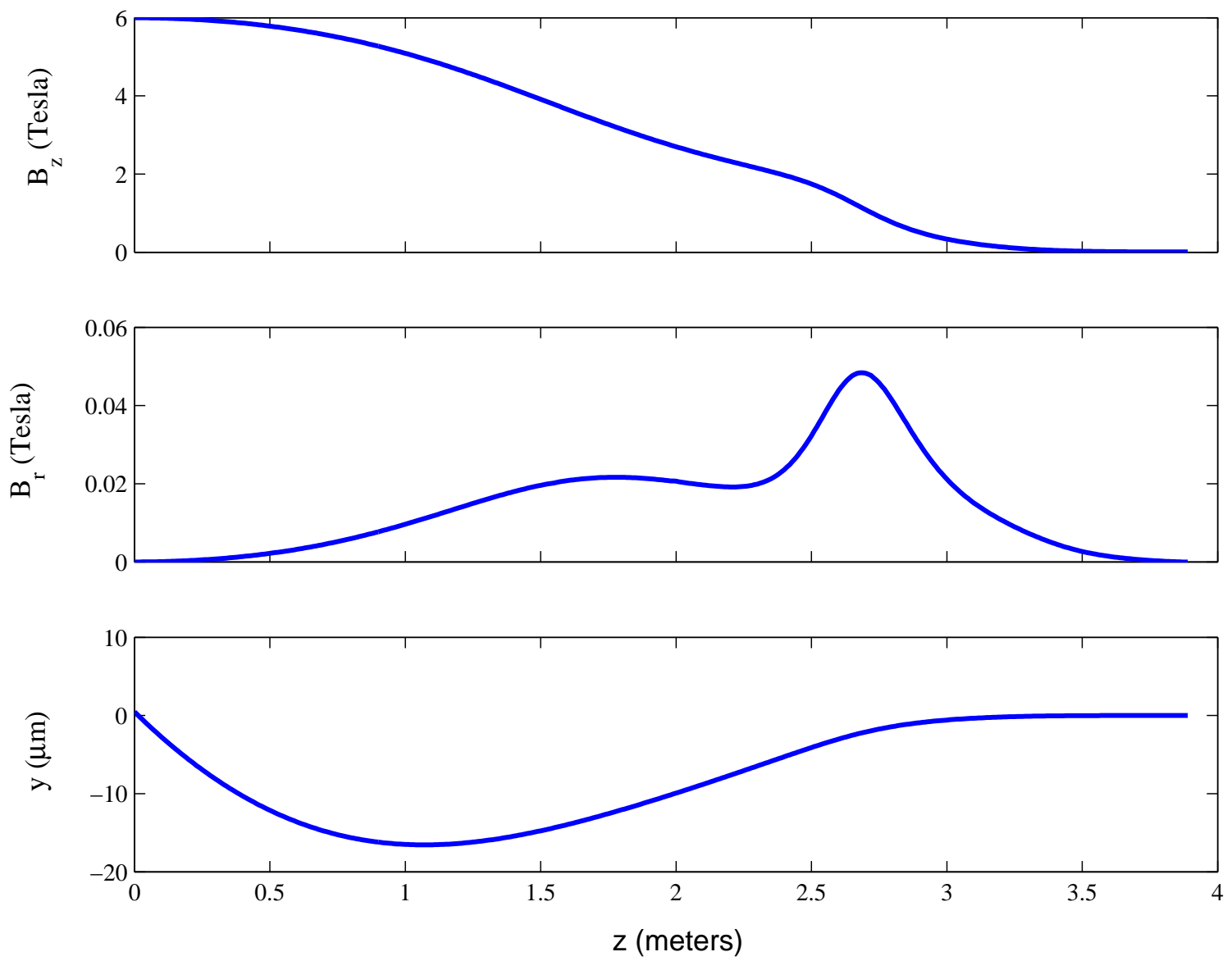

FIG. 4: Magnetic field in prototype Linear Collider Detector solenoid along the beam trajectory: (a) longitudinal field; (b) radial field; (c) resulting vertical deflection of a $500 \mathrm{GeV}$ beam within the solenoid. 

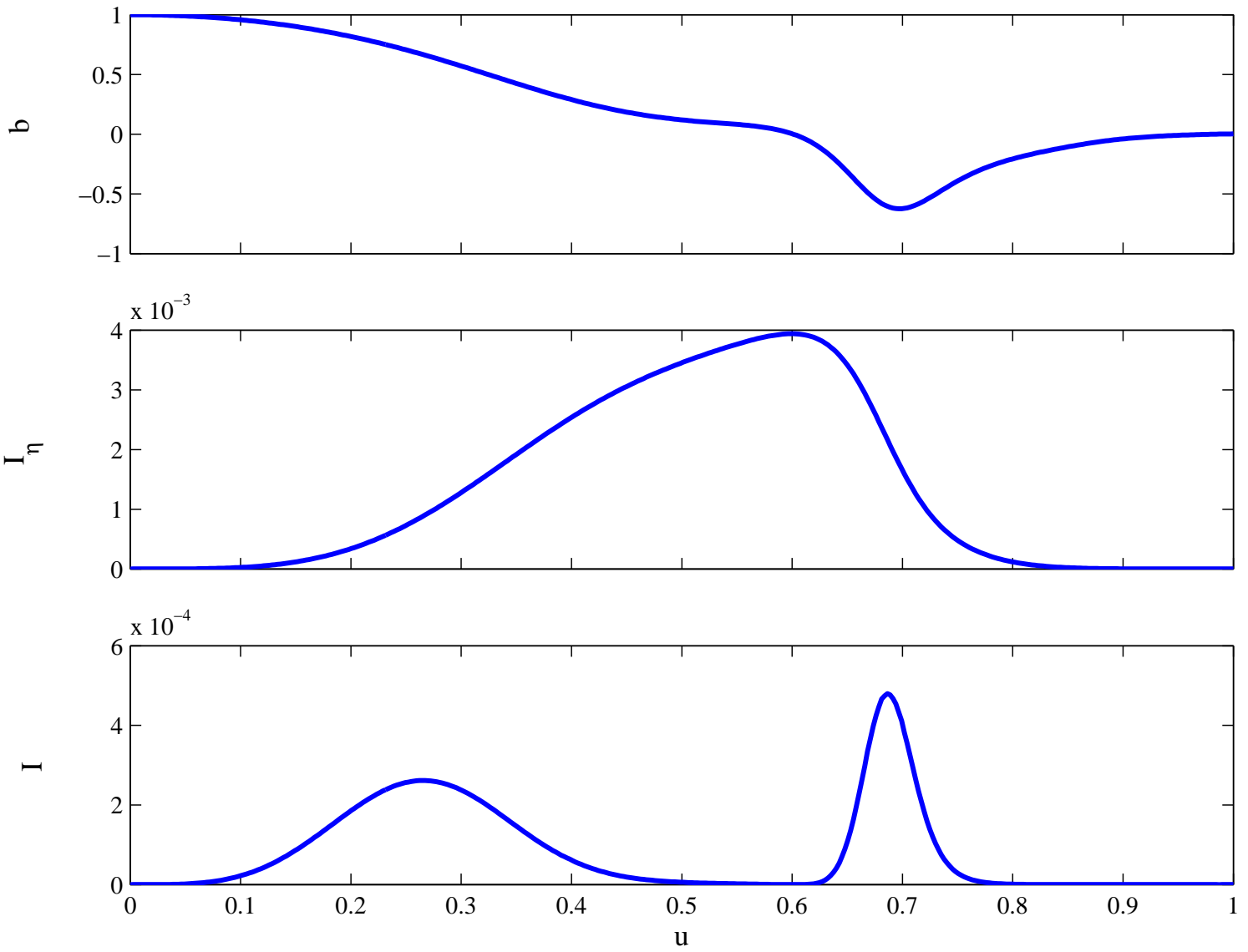

FIG. 5: Functions related to vertical beam size growth from quantum fluctuations in synchrotron radiation: (a) Normalized deflecting field $b$; (b) dispersion integral $I_{\eta}$; (c) Integrand of Equation 15, $I$, as defined in the text. 